\title{
Política pública en materia de fomento gubernamental. Determinantes de la demanda turística de 30 destinos turísticos en México Mario Alberto Velázquez García ${ }^{1}$ y Hugo Nathanael Lara Figueroa ${ }^{2}$
}

\footnotetext{
${ }^{1}$ Profesor Investigador- El Colegio del Estado de Hidalgo SNI I, mvelazquez@elcolegiodehidalgo.edu.mx

${ }^{2}$ Profesor Investigador Tecnológico de Estudios Superiores Ectaepec, SNI C
}

Recibido: 15-11-18 Aceptado: 19-02-2019 Publicado: 15-08-2019

Cómo citar este artículo: Velázzquez M y Lara H (2019) "Política pública en materia de fomento guberamental. Determinantes de la demanda turística de 30 destinos turísticos en México" en Revista Científica de Estudios Urbano Regionales HatsöHnini, Año 1, Núm. 1, Abril-Septiembre 2019, págs. 23-38 México

DOI: https://doi.org/10.47386/2019V1N1A3

\section{Resumen}

El presente trabajo tiene como objetivo principal determinar estrategias para el fomento gubernamental del turismo, para ello se generó un modelo econométrico que nos permitiera identificar los factores que explican el comportamiento de la demanda turística de 30 destinos de México en el periodo que abarca de 2000 a 2013. Se estimó un modelo de regresión múltiple general utilizando información de diversas fuentes oficiales de turismo e INEGI. Se encontró que la infraestructura en términos de alojamiento y servicios recreativos y de

o cio tienen una incidencia en el comportamiento de la demanda turística de los destinos analizados. Los resultados obtenidos sugieren la implementación de estrategias vinculadas al fomento turístico en agencias de viajes, hoteles, y bares considerando las características de cada tipo de destino. El documento cuenta con la siguiente estructura. En la primera sección se realiza una introducción sobre la relevancia que tiene el turismo a nivel nacional e internacional en términos de crecimiento $\mathrm{y}$ desarrollo económico. En la segunda se propone una definición sobre fomento al turismo gubernamental. En la tercera sección se describen y especifican las variables incorporadas al modelo $y$ se estima el modelo de regresión, así como la simulación de escenarios para conocer la incidencia que tienen los factores en la variable de interés (llegada de turistas).
Finalmente, el documento cierra con un apartado de conclusiones y estrategias de política de fomento al turismo.

\section{Introducción}

El interés que existe entre los gobiernos, el sector privado, y organizaciones sociales sobre el turismo, se explican por la creciente cantidad de personas, recursos económicos y transformaciones sociales y culturales relacionadas al desarrollo mismo de esta actividad y su constante crecimiento. El turismo es la única actividad económica que no ha dejado de crecer desde finales de la Segunda Guerra Mundial, pasando de 25 millones de turistas a nivel mundial en el año de 1950, a 278 millones en 1980. Para el año 1995 existieron 527 millones de turistas en el mundo que en el año 2014 subieron a 1.133 millones. Por su parte los ingresos totales de todos los destinos turísticos fueron de 2.000 millones de dólares en 1950, para el año de 1995 esta cifra llegó a los 104.000 millones y para 2014 llegaron a 1.425 .00 millones (OMT, 2015).

México cuenta con una serie de ventajas comparativas (localización geográfica, productos turísticos naturales y culturales, potencial de conectividad) sobre el turismo (Sectur, 2013). No obstante el país ha perdido participación en el mercado internacional. México ocupó en el año 2014 el décimo lugar mundial por llegadas de turistas internacionales, lo que represento el arribo de 29.3 millones de personas. Pero en lo que respecta a los ingresos obtenidos por las divisas internacionales el país ocupa la posición número 22 en el ranking mundial, $16.2 \mathrm{mil}$ millones de dólares. 


\section{Política pública en materia de fomento gubernamental}

Finalmente, México ocupa el lugar número 30 de la clasificación de la OMT en gasto en turismo internacional: 9,6 mil millones de dólares. En el 2014, algunos de los destinos turísticos emergentes que han rebasado a México en captación de divisas por turismo son Macao (50.5 mil millones de dólares), Tailandia (38.4 mil millones de dólares) y Hong Kong (38.4 mil millones de dólares) (OMT, 2015).

El turismo a nivel mundial continua presentando un ritmo de crecimiento sostenido, en el periodo de los años 2000 al 2012 lo hizo a un promedio de 3.6 por ciento anual. En contraste el crecimiento de esta actividad en México presenta un ritmo de crecimiento moderado. En el periodo 1980 a 2012 la tasa de crecimiento de llegadas de turistas internacionales fue de 2 por ciento anual. En comparación, entre el año 2000 al 2012 existen zonas turísticas emergentes que crecen a tasas más elevadas: Turquía (11.6\%), Hong Kong (8.6\%), Malasia (7.8\%), Japón (4.8\%) y Rusia, mientras México creció $1 \%$ en el mismo periodo de tiempo (OMT, 2015). Los siguientes resultados son parte de una investigación financiada con el Fondo Sectorial Sectur (2014). E1 proyecto tenía el título de: "Metodología para la planeación de políticas públicas de Fomento y promoción del sector turístico de México". Uno de los objetivos generales de la investigación y de este trabajo, era proponer propuestas de políticas de fomento al turismo. Uno de los métodos de investigación utilizados y que aquí retomamos, fue un modelo matemático que nos permitía identificar los diferentes impactos que tienen las variables relacionadas a la infraestructura turística disponible en los rubros de alojamiento, servicios, actividades recreativas y de ocio. Al respecto se consideraron datos en una serie de tiempo que va del año 2000 a 2013 , con lo que resultó posible determinar no sólo la evolución de las variables seleccionadas, sino también el impacto generado en los últimos años sobre la actividad turística en México.

El siguiente estudio está basado en 30 localidades que el gobierno federal mexicano ha definido como prioritarias en materia de políticas de fomento al turismo. Esta son: 1) Acapulco; 2) Cancún; 3) Rivera Maya; 4)

Veracruz -Boca del Río; 5) Mazatlán; 6) Puerto Vallarta; 7) Los Cabos; 8) Nuevo Vallarta; 9) Ensenada; 10) IxtapaZihuatanejo; 11) Manzanillo; 12) Cozumel; 13) Puebla; 14) León; 15) Mérida; 16) Querétaro; 17) Oaxaca; 18) San Juan de los Lagos; 19) Morelia; 20) Tuxtla Gutiérrez; 21) San Luis Potosí; 22) Cuernavaca; 23) Villahermosa; 24) Hermosillo; 25) Xalapa; 26) Guanajuato; 27) Aguascalientes; 28) Zacatecas; 29) Ciudad Juárez; 30) Tijuana (INEGI, 2000-2014).

\section{Fomento al turismo gubernamental}

Como el objetivo central de este artículo es proporcionar una serie de propuestas de políticas que puedan incentivar la actividad turística en una localidad mediante la actividad gubernamental, presentamos de manera sintética el concepto de política que guía nuestra propuesta: el fomento al turismo. 
La relevancia de este radica en el hecho de que existen pocos intentos por definir esta actividad gubernamental, a pesar de la importancia que ha tomado en la planeación turística en México.

\section{Concepto de fomento}

El fomento al turismo hace referencia a las organizaciones y actividades del gobierno a través de la cuales se busca incentivar la oferta turística con el fin de generar un aumento, mejora o diversificación de las instalaciones que facilitan o permiten a los turistas completar el ciclo de un viaje turístico: transporte para llegar de su hogar al destino, satisfacer sus necesidades de hospedaje y comida, uso o disfrute de los paisajes o servicios que motivaron el viaje, resolver eventualidades (médicas, legales, entre otras) y regresar a su lugar de partida.
Dicho de otro modo, el fomento al turismo podemos definirlo como la actividad de protección, auxilio o impulso que el gobierno brinda para el desarrollo de la actividad turística en una localidad específica, región o país. Las actividades de éste serán una amplia gama de medidas que buscan estimular el crecimiento de la actividad turística en un espacio social determinado; el fomento implica la participación de diversas dependencias gubernamentales para el logro de un mismo objetivo: generar las condiciones físicas, económicas, fiscales, culturales y sociales que permitan el desarrollo de un conjunto de interacciones y prácticas consideradas como turísticas dentro de un espacio social determinado.

El concepto de fomento al turismo es entonces, todas aquellas actividades del gobierno destinadas a la construcción, -

\section{Cuadro No. 1}

\begin{tabular}{|ll|}
\hline \multicolumn{1}{|c|}{ Dimensiones del FTG } \\
\hline 1. & $\begin{array}{l}\text { Aumentar los ingresos del gobierno mediante una mayor entrada de divisas o un } \\
\text { incremento en las empresas que pagan impuestos }\end{array}$ \\
\hline 2. & $\begin{array}{l}\text { Proporcionar información que oriente y motive a los posibles consumidores para } \\
\text { el uso de un espacio turístico (los atractivos, infraestructura y servicios con los que } \\
\text { cuenta }\end{array}$ \\
\hline 3. & Cambios en el uso y la percepción de los individuos hacia un espacio \\
\hline 4. & $\begin{array}{l}\text { Transformar la especialización económica de un lugar o la economía nacional o } \\
\text { regional }\end{array}$ \\
\hline 5. & $\begin{array}{l}\text { Reconfigurar la relación de los actores locales donde grupos de escasos recursos } \\
\text { obtengan nuevas formas de ascenso social }\end{array}$ \\
\hline 6. & $\begin{array}{l}\text { Transformar la percepción sobre un lugar, ciudad o país buscando no sólo atraer } \\
\text { turistas, sino que el gobierno sea percibido de una mejor forma por la población } \\
\text { nacional e internacional }\end{array}$ \\
\hline
\end{tabular}

Fuente: Elaboración propia 
Cuadro No. 2

\begin{tabular}{|c|c|}
\hline \multicolumn{2}{|c|}{ Desafios del Fomento al Turismo Gubernamental } \\
\hline $\begin{array}{l}\text { 1. Espacial } \\
\text { 2. Político }\end{array}$ & $\begin{array}{l}\text { Selección de lugares o regiones que son } \\
\text { consideradas como poseedoras del potencial de } \\
\text { contener y desarrollar actividades turísticas. } \\
\text { El gobierno debe elegir cuáles serán los } \\
\text { actores, proyectos y lugares que serán } \\
\text { apoyados para el desarrollo de proyectos } \\
\text { turísticos. Esta selección no se produce } \\
\text { exclusivamente bajo criterios técnicos, sino } \\
\text { que tiene lugar dentro de un entorno político } \\
\text { donde diversos actores gubernamentales, } \\
\text { políticos profesionales, pero también } \\
\text { compañías privadas nacionales y extranjeras, } \\
\text { así como organizaciones civiles buscan incidir } \\
\text { en las decisiones de política gubernamental de } \\
\text { inversión }\end{array}$ \\
\hline 3. Coordinación & $\begin{array}{l}\text { a) las distintas posturas y objetivos que las } \\
\text { agencias y niveles de gobierno (federal, estatal } \\
\text { y municipal) tienen sobre la actividad turística. } \\
\text { b) divergencias entre agencias se presenta } \\
\text { en las zonas fronterizas, donde los gobiernos } \\
\text { tendrán disposiciones legales y administrativas } \\
\text { distintas para regular la operación turística. } \\
\text { c) los organismos internacionales, los } \\
\text { cuales pueden contener directivas distintas } \\
\text { sobre eldesarrollo de la actividad turística y las } \\
\text { regulaciones locales, regionales o nacionales } \\
\text { Los planes de inversión son construidos a partir } \\
\text { de restricciones de tiempo para los distintos } \\
\text { actores involucrados; los gobiernos locales } \\
\text { tienen una duración menor que los gobiernos } \\
\text { estatales y municipales, por ello requieren de } \\
\text { obtener resultados más inmediatos }\end{array}$ \\
\hline
\end{tabular}

Fuente: Elaboración propia 
conservación y mantenimiento de la infraestructura turística, que abarca desde carreteras, construcción de hoteles, pero también servicios complementarios (bancos, centros comerciales, servicio telefónico), así como el mantenimiento y difusión de las actividades culturales (museos, talleres, exposiciones, grupos artísticos). Al centrarnos en la promoción como una actividad de gobierno buscamos resaltar que estas actividades no tienen únicamente una finalidad económica, sino que existen intereses políticos, culturales y sociales relacionados.

\section{Modelo Econométrico}

Existe una extensa literatura que ha abordado el comportamiento del sector turístico tanto a nivel nacional como internacional. De manera esquemática podemos destacar 2 modelos de análisis: los modelos econométricos lineales y logísticos con información agregada y desagregada, y el denominado ciclo de vida de los destinos turísticos (CVDT) donde se utilizan correlaciones y regresiones, así como el método de casos. Con respecto a los primeros el modelo agregado se ha desarrollado a partir del enfoque neoclásico de elección donde la demanda turística se comporta de la misma manera que otros bienes, de tal manera que un turista es un individuo -racional- que, con base en sus recursos disponibles, restricciones presupuestarias, precio de otros bienes en cuestión, así como sus gustos y preferencias, elige entre la actividad turística y otras actividades o bienes, tratando de maximizar su utilidad.
En resumen, los modelos pretenden predecir no sólo las variables que impactan en la decisión de un individuo u hogar cuando opta por salir de viaje o no, sino también los factores que determinan la elección de un destino turístico.

Este tipo de modelos se encuentran basados en la Teoría de las Características de Lancaster perfeccionados posteriormente por Rugg y Morley generando los denominados modelos de elección modal distribuidos en 2 etapas: la primera que consiste en predecir las variables que inciden en la elección de salir o no salir; y la segunda, estima probabilidades en la elección del tipo de destino (Lancaster, 1966; Morley, 1992).

La popularidad de los modelos de elección discreta se debe en gran parte al trabajo de McFadden ya que intenta explicar el comportamiento individual considerando como variables explicativas las características socio económicas de los individuos y los atributos de las alternativas (características de cada destino). Si bien este tipo de modelización es más completo en comparación al anterior, una gran limitación tiene que ver con la falta de disponibilidad de información de variables tanto de los individuos, como de los atributos de los lugares (McFadden, 1973).

\section{Evidencia empírica}

Existen un sinnúmero de estudios que han utilizado los diferentes modelos teórico-empíricos antes mencionados. Entre ellos podemos destacar el modelo desagregado realizado por Rodríguez y 
y compañeros donde, mediante un modelo logístico multinomial, se identificaron los atributos que los turistas consideran durante su estancia e n las Is las Canarias de España (Rodríguez Feijoo, Dávila Quintana, \& Rodríguez Caro, 2000).

Para el caso mexicano destaca el modelo desarrollado por Guzmán y otros en 2011 en el cual se determinó la relación directa que la demanda turística tiene con el comportamiento de la economía canadiense y de Estados Unidos, así como con la promoción que un turista da a los futuros visitantes (Guzmán Soria, Garza Carranza, Rebollar, Hernández Martínez, \& García Salazar, 2011).

Para el caso del presente estudio, se estimó un modelo de regresión lineal múltiple y se simularon cambios en la variable llegada de turistas ante cambios en las variables que mostraron mayor incidencia en su comportamiento.

\section{Marco muestral}

Con el fin de identificar y priorizar las necesidades de fomento, mediante el desarrollo de una interpretación explicativa de las variaciones existentes en los treinta destinos turísticos, se utilizó información disponible en términos de fomento turístico de acuerdo a las matrices de indicadores de todos los programas coordinados por el Fondo Nacional de Fomento al Turismo (FONATUR), por lo que el modelo incluye únicamente información sobre infraestructura turística (tomada desde el punto de vista de "fomento turístico") en términos de hospedaje y alojamiento
(Hoteles, Moteles, y Casas de Huéspedes), ocio y esparcimiento (Bares, Discotecas y Centros Nocturnos, Restaurantes, Cafeterías), así como de servicios turísticos (Agencias de Viajes) para los 30 destinos especificados. Con dichas variables se explicó, mediante la predicción de cambios en las variables independientes (relacionadas a la promoción y fomento), el impacto que éstas tienen en la llegada de turistas (Sectur, 2015). Para la estimación del modelo se empleó la regresión lineal múltiple, la cual permite identificar los posibles impactos de las variables dependientes respecto a la independiente; el modelo de la demanda turística, expresada en el número de turistas que arriban a los destinos en México, quedó formalmente especificado en:

THEH $H_{8,30}=\beta_{0}+\beta_{1} * A V_{-} 3+\beta_{2} * R E S T_{\_} 3$

$+\beta_{3} * H_{-} 3+\beta_{4} * M_{-} 3+\beta_{5} * O H_{-} 3+\beta_{6}$

$* C A F_{-} 3+\beta_{7} * D C N \_3+\beta_{8} * B A R_{-} 3+U I$

Donde:

- THEH: Variable dependiente que representa el número de turistas que arriban a las localidades del año $2000 \mathrm{al}$ 
- 8,30: Indica las 8 variables que explican el modelo, mientras que 30 es el total de observaciones registradas equivalentes a las localidades representativas del país.

- AV_3: Indica la cantidad de Agencias de Viajes registradas del año 2000 al 2013 en las 30 localidades.

- REST 3: Indica la cantidad de Restaurantes registrados del año 2000 al 2013 en las localidades.

- H 3: Indica la cantidad de Hoteles registrados del año 2000 al 2013 en las localidades.

- M_3: Indica la cantidad de Moteles registrados del año 2000 al 2013 en las localidades.
- OH 3: Indica la Ocupación Hotelera registrada del año 2000 al 2013 en las localidades.

- CAF_3: Indica el total de Cafeterías registradas del año 2000 al 2013 en las localidades.

- DCN 3: Indica el total de Discotecas y Centros Nocturnos registrados del años 2000 al 2013 en las localidades.

- BAR_3: Indica el total de Bares registrados del año 2000 al 2013 en las localidades.

- $\beta 0$ : Indica el intercepto de la ecuación $\beta 1, \beta 2, \beta 3, \beta 4$.

- $\beta 1, \beta, \beta 3, \beta 4$, Indican los estimadores del modelo a través de M.C.O.

Cuadro No. 3

Localidades consideradas en el modelo

LUGARES SELECCIONADOS

\begin{tabular}{l|l}
\hline Acapulco & Morelia \\
Aguascalientes & Nuevo Vallarta \\
Cancún & Oaxaca \\
Ciudad Juárez & Puebla \\
Cozumel & Puerto Vallarta \\
Cuernavaca & Querétaro \\
Ensenada & Rivera Maya \\
Guanajuato & San Juan de los \\
Hermosillo & Lagos \\
Ixtapa- Zihuatanejo & San Luis Potosí \\
León & Tijuana \\
Los cabos & Tuxtla Gutiérrez \\
Manzanillo & Veracruz- Boca del \\
Mazatlán & Río \\
Villahermosa & Xalapa \\
& Zacatecas \\
\hline
\end{tabular}

Fuente: Elaboración propia 
Respecto a las especificaciones de las variables incluidas se consideró como variable dependiente (Y) al número de Turistas que arribaron al lugar, mientras que las variables predictoras (X; independientes) corresponden a: Agencias de Viajes, Bares, Discotecas y Centros Nocturnos, Restaurantes, Cafeterías, Hoteles, Moteles y Ocupación Hotelera, obtenidas del Anuario Estadístico y Geográfico:

\begin{tabular}{|c|c|c|c|}
\hline \multicolumn{4}{|c|}{ Cuadro No. 4} \\
\hline VARIABLE & $\begin{array}{l}\text { UNIDAD DE } \\
\text { MEDIDA }\end{array}$ & $\begin{array}{c}\text { TIPO DE } \\
\text { VARIABLE }\end{array}$ & FUENTE DE INFORMACIÓN \\
\hline $\begin{array}{c}\text { Turistas que } \\
\text { arriban al lugar } \\
\text { (nacionales y } \\
\text { extr anjer os) }\end{array}$ & $\begin{array}{l}\text { Número de personas } \\
\text { que arribaron a la } \\
\text { localidad al año }\end{array}$ & Discreta & $\begin{array}{l}\text { INEGI, Anuario Estadístico y } \\
\text { Geográfico de las diferentes } \\
\text { entidades }\end{array}$ \\
\hline ocupación hotelera & $\begin{array}{c}\text { Porcentaje de } \\
\text { ocupación hotelera } \\
\text { anual }\end{array}$ & Discreta & $\begin{array}{l}\text { INEGI, Anuario Estadístico y } \\
\text { Geográfico de las diferentes } \\
\text { entidades }\end{array}$ \\
\hline Hoteles & $\begin{array}{l}\text { Número de hoteles } \\
\text { registrados al año }\end{array}$ & Discreta & $\begin{array}{l}\text { INEGI, Anuario Estadístico y } \\
\text { Geográfico de las diferentes } \\
\text { entidades }\end{array}$ \\
\hline Moteles & $\begin{array}{l}\text { Número de moteles } \\
\text { registrados anualmente }\end{array}$ & Discreta & $\begin{array}{l}\text { INEGI, Anuario Estadístico y } \\
\text { Geográfico de las diferentes } \\
\text { entidades }\end{array}$ \\
\hline Restaurantes & $\begin{array}{l}\text { Número de restaurantes } \\
\text { registrados anualmente }\end{array}$ & Discreta & $\begin{array}{l}\text { INEGI, Anuario Estadístico y } \\
\text { Geográfico de las diferentes } \\
\text { entidades }\end{array}$ \\
\hline Cafeterías & $\begin{array}{l}\text { Número de cafeterías } \\
\text { registradas al año }\end{array}$ & Discreta & $\begin{array}{l}\text { INEGI, Anuario Estadístico y } \\
\text { Geográfico de las diferentes } \\
\text { entidades }\end{array}$ \\
\hline Bares & $\begin{array}{l}\text { Número de bares } \\
\text { registrados al año }\end{array}$ & Discreta & $\begin{array}{l}\text { INEGI, Anuario Estadístico y } \\
\text { Geográfico de las diferentes } \\
\text { entidades }\end{array}$ \\
\hline $\begin{array}{c}\text { Discotecas y } \\
\text { centros nocturnos }\end{array}$ & $\begin{array}{l}\text { Número de discotecas } \\
\text { y centros nocturnos } \\
\text { registrados anualmente }\end{array}$ & Discreta & $\begin{array}{l}\text { INEGI, Anuario Estadístico y } \\
\text { Geográfico de las diferentes } \\
\text { entidades }\end{array}$ \\
\hline Agencias de viajes & $\begin{array}{l}\text { Número de agencias de } \\
\text { viajes registradas al } \\
\text { año }\end{array}$ & Discreta & $\begin{array}{l}\text { INEGI, Anuario Estadístico y } \\
\text { Geográfico de las diferentes } \\
\text { entidades }\end{array}$ \\
\hline
\end{tabular}

Fuente: Elaboración propia 
Luego de la implementación de los modelos en sus cuatro formas, se compararon los resultados y se optó por el modelo con variables elevadas al cubo, dado que éste obtuvo un mejor ajuste con respecto al comportamiento de la variable de interés, así como al poder explicativo del modelo final.

\section{Análisis descriptivo de las variables de interés en el modelo}

Para realizar la estimación econométrica del modelo se consideró conveniente realizar en primera instancia un análisis estadístico que permitiera mostrar la tendencia de la información disponible del periodo de estudio (2000-2013). Como se observa, en la mayoría de los casos, la desviación de las observaciones no supera al promedio y al punto intermedio, lo cual indica que la mayoría de los datos se localizan alrededor de estos valores, con excepción de las variables de Discotecas y Centros Nocturnos y Moteles. Por otro lado, de las ocho variables seleccionadas (Turistas que se hospedaron en establecimientos de hospedaje, Agencias de Viajes y Discotecas y Centros Nocturnos, muestran, según el apuntamiento de la gráfica, una gran concentración de los datos, mientras que para los casos de Bares, Restaurantes, Cafeterías, Hoteles, Moteles y Ocupación Hotelera), se observó una baja concentración de los datos; lo que se confirma con los valores arrojados por la curtosis.

En términos de asimetría, se observó que en todos los casos el promedio es mayor al punto intermedio, es decir, la mediana no supera a la media; por lo tanto, existe una asimetría positiva, o, en otras palabras, los datos se encuentran distribuidos a la izquierda del histograma. Al realizar el análisis descriptivo se observa que durante el periodo de estudio el país ha recibido un promedio de 998 mil 282 turistas que se hospedaron en los diferentes establecimientos de hospedaje; existen casi 60 agencias de viajes, 40 bares, 11 discotecas y centros nocturnos, 226 restaurantes, 20 cafeterías, 106 hoteles, 10 moteles y un porcentaje de

Cuadro No. 5

Estadística descriptiva del modelo general

\begin{tabular}{|c|c|c|c|c|c|c|c|c|c|}
\hline \multicolumn{10}{|c|}{ ESTADÍSTICOS } \\
\hline & $\begin{array}{l}\text { Turistas que se } \\
\text { hospedaron en } \\
\text { establecimientos } \\
\text { de hospedaje }\end{array}$ & $\begin{array}{l}\text { Agencias } \\
\text { de viajes }\end{array}$ & Bares & $\begin{array}{c}\text { Discotecas y } \\
\text { centros } \\
\text { nocturnos }\end{array}$ & Restaurantes & Cafeterías & Hoteles & Moteles & $\begin{array}{c}\text { Ocupación } \\
\text { hotelera }\end{array}$ \\
\hline Media & $998,282.56$ & 59.94 & 40.51 & 11.67 & 226.29 & 20.30 & 106.04 & 10.76 & 50.699 \\
\hline Mediana & $732,013.00$ & 45.00 & 30.00 & 8.00 & 178.00 & 15.00 & 93.00 & 6.50 & 50.275 \\
\hline $\begin{array}{l}\text { Desviación } \\
\text { estándar }\end{array}$ & 890187.727 & 53.436 & 38.603 & 13.133 & 176.905 & 17.497 & 61.503 & 12.562 & 11.4966 \\
\hline Asimetría & 2.369 & 1.652 & 1.589 & 2.607 & .989 & .817 & .858 & 1.304 & -.023 \\
\hline Curtosis & 6.662 & 3.996 & 2.117 & 8.532 & .386 & -.152 & .249 & .984 & .567 \\
\hline
\end{tabular}

Fuente: Elaboración propia con información del INEGI, 2000-2013 


\section{Estimación e interpretación de los resultados}

Para la estimación del modelo se empleó el método de Mínimos Cuadros Ordinarios (M.C.O), a través del software SPSS Statistics versión 23. Debido a que en la mayoría de los casos se presentaron bajos niveles de correlación (lo cual sugiere que la rela - ción entre las variables empleadas no muestra un comportamiento lineal), se ajustó el modelo mediante la incorporación de las variables en forma cuadrática y cúbica, ello con el fin de mejorar las estimaciones obtenidas para la elaboración de pronósticos.

Cuadro No. 6

Resultados de la estimación del modelo general

COEFICIENTES

\begin{tabular}{|c|c|c|c|c|c|}
\hline \multicolumn{6}{|c|}{$\begin{array}{l}\text { VARIABLE DEPENDIENTE: THEH (TURISTAS QUE SE HC } \\
\text { ESTABLECIMIENTOS DE HOSPEDAJE) }\end{array}$} \\
\hline \multirow[t]{17}{*}{ MODELO } & & \multicolumn{2}{|c|}{ Coeficientes } & \multirow[t]{2}{*}{$\mathrm{t}$} & \multirow[t]{2}{*}{ Sig. } \\
\hline & & B & $\begin{array}{l}\text { Error } \\
\text { estándar }\end{array}$ & & \\
\hline & (Constante) & 443596.849 & 62449.047 & 7.103 & .000 \\
\hline & $\mathrm{AV} \_3$ & .027 & .008 & 3.363 & .001 \\
\hline & REST_3 & .005 & .001 & 7.780 & .000 \\
\hline & H_3 & .150 & .015 & 10.023 & .000 \\
\hline & M_3 & -4.337 & 1.437 & -3.018 & .003 \\
\hline & $\mathrm{OH} \_3$ & 1.394 & .414 & 3.370 & .001 \\
\hline & CAF_3 & -4.501 & .516 & -8.718 & .000 \\
\hline & DCN_3 & -4.651 & .514 & -9.048 & .000 \\
\hline & BAR_3 & .228 & .060 & 3.823 & .000 \\
\hline & $\mathrm{R}$ & $.919^{\mathrm{a}}$ & & & \\
\hline & $\mathrm{R}$ cuadrado & .844 & & & \\
\hline & $\begin{array}{l}\text { R cuadrado } \\
\text { ajustado }\end{array}$ & .835 & & & \\
\hline & $\mathrm{F}$ & 91.408 & & & \\
\hline & Probabilidad (F) & $.000^{\mathrm{b}}$ & & & \\
\hline & Durbin-Watson & 2.042 & & & \\
\hline
\end{tabular}

A. Y B. PREDICTORES: (CONSTANTE), BAR_3, M_3, AV_3, CAF_3, OH_3, H_3, DCN_3, REST_3

Fuente: Elaboración propia. Salidas de SPSS Statistics 


\section{Interpretación de resultados}

Los resultados de la estimación son considerados adecuados en términos de multicolinealidad ( $r$ ajustado), autocorrelación (Durbin Watson) y heterocedasticidad (Estadístico F y su Probabilidad). Todas las variables explicativas presentan un impacto estadísticamente significativo (significancia); mientras que el modelo explica en 84 por ciento el comporta- miento de la variable de interés. Lo anterior significa que el modelo obtenido es confiable, y que el 84 por ciento de los cambios que se presentan en la llegada de turistas son atribuibles a las variables empleadas durante la estimación. A nivel global, la estimación es estadísticamente significativa (.000), con lo que se rechaza la hipotesis nula de no impacto significativo lineal sobre la variable dependiente.

\section{Cuadro No. 7}

Prueba de Kolmogorov-Smirnov para conocer el supuesto de distribución normal de los errores

\begin{tabular}{|c|c|c|c|}
\hline HIPÓTESIS NULA & PRUEBA & SIG. & DECISIÓN \\
\hline $\begin{array}{l}\text { La distribución de unstandardized residual es } \\
\text { normal con la media }-0.0000 \text { y la desviación } \\
\text { estándar } 299,470.963\end{array}$ & $\begin{array}{l}\text { Prueba de Kolmogorov- } \\
\text { Smirnov para una } \\
\text { muestra }\end{array}$ & $.200^{1 / 2}$ & $\begin{array}{l}\text { Rechace la } \\
\text { hipótesis nula }\end{array}$ \\
\hline \multicolumn{4}{|c|}{ Se muestran significaciones asintónicas. El nivel de significación es .05 } \\
\hline${ }^{1}$ Liliefors corregido & & & \\
\hline
\end{tabular}

Fuente: Elaboración propia con base en datos del INEGI, 2000-2013

Cuadro No. 8

Estadísticos de colinealidad del modelo general

COEFICIENTES ${ }^{A}$

\begin{tabular}{|c|c|c|}
\hline \multirow{3}{*}{ (CONSTANTE) } & \multicolumn{2}{|c|}{$\begin{array}{c}\text { Estadísticas de } \\
\text { colinealidad }\end{array}$} \\
\hline & Tolerancia & VIF \\
\hline & & \\
\hline$A V \_3$ & .779 & 1.284 \\
\hline REST_3 & .180 & 5.560 \\
\hline H_3 & .663 & 1.509 \\
\hline M_3 & .648 & 1.542 \\
\hline OH_3 & .716 & 1.396 \\
\hline CAF_3 & .558 & 1.792 \\
\hline DCN_3 & .536 & 1.866 \\
\hline BAR_3 & .233 & 4.290 \\
\hline
\end{tabular}

Fuente: Elaboración propia con base en datos del INEGI, 2000-2013 
La distribución normal de los errores y varianza de los residuales se determinó a través de la prueba de KolmogorovSmirnov y su juego de hipótesis donde se rechazó la hipótesis nula sobre la distribución normal de los residuos estandarizados.

Una vez realizadas las pruebas así como los niveles de ajuste, los resultados muestran que el número de turistas que se hospedan en establecimientos de hospedaje se relaciona positivamente con las agencias de viajes, los restaurantes, los hoteles, los bares y la ocupación hotelera, siendo las agencias de viajes, la disponibilidad de hoteles y bares en los lugares de destino las variables que más inciden en la llegada de turistas. Por su parte, la infraestructura existente en términos de moteles, discotecas y centros nocturnos y las cafeterías inciden de manera negativa en la variable de interés.

\section{Interpretación de los resultados}

Con respecto a la interpretación de resultados podemos decir que la disponibilidad servicios de agencias, hoteles y la existencia de espacios de ocio y diversión tales como bares son atributos que inciden en que un turista opte por acudir a los destinos analizados. Caso contrario, la disponibilidad de moteles, discotecas y centros nocturnos, así como cafeterías afectan negativamente la llegada de turistas.Si bien estos resultados de las variables que guardan una asociación negativa con la variable de interés podrían considerarse contraintuitivos, lo anterior no significa necesariamente que la inversión en este tipo de infraestructura implica una caída en la llegada de turistas, antes bien sugiere que, dadas las características de los hoteles disponibles, las personas optan no tanto por sitios donde existan moteles o cafeterías por mencionar un ejemplo, sino espacios o lugares de alojamiento donde ya se encuentran incluidos este tipo de servicios e infraestructura, situación muy habitual para los destinos de playa. Dicho de otro modo, los valores negativos reflejan la fuerte preferencia de los turistas por estancias de hospedaje específica (hotelera) donde servicios como cafeterías ya se encuentran incluidos en éstos.

A fin de facilitar la interpretación de los resultados se estimaron valores hipotéticos de los cambios en la llegada de turistas ante cambios en las variables agencias, hoteles y bares, manteniendo constantes el resto de las variables. Para ello, se pronosticó cómo cambia el arribo de turistas cuando aumenta la infraestructura disponible de cada una de las variables antes mencionadas.

Tal y como se observa en la gráfica anterior, el aumento o la existencia de agencias de viajes y hoteles incrementan de manera considerable el arribo de turistas a los destinos incorporados en el modelo, lo cual significa que éste tipo de bienes funcionan como incentivos para hacer que muchos turistas opten por acudir a lugares donde no existen complicaciones para encontrar alojamiento, pero también, donde quienes deciden pasar su estancia en nuestro país, pueden disponer de agencias que facilitan los procesos de - 
adquisición de vuelos o transporte terrestre, así como de recorridos o eventos en cada lugar. Adicionalmente y considerando que una parte del turismo se realiza con fines de esparcimiento, ocio y recreación, resulta importante también la existencia de bares o sitios donde la disponibilidad de establecimientos para consumir alimentos es adecuada.

\section{Estrategias de acción para el diseño de las políticas de fomento de turismo}

Resultado del modelo proponemos una serie de estrategias de acción generales para el diseño de las políticas de fomento al turismo en México:
Estandarización de la calidad de las instalaciones turísticas, seguridad e higiene. Una línea de acción para la operación de las políticas públicas es una transformación y mejora en la regulación federal sobre los servicios turísticos y los programas de apoyo para la mejora de las instalaciones y la capacitación. Como podemos ver este estudio nos permitió determinar que el número de turistas que arriban a las localidades de playa se relaciona positivamente con las variables de Discotecas y Centros Nocturnos, Establecimientos de Alimentos y B e bid a s, a sí com o con los establecimientos de Hospedaje de 5, 4 y 2 estrellas.

Figura No. 1

Incremento en la llegada de turistas antes cambios en infraestructura y servicios de los destinos turísticos

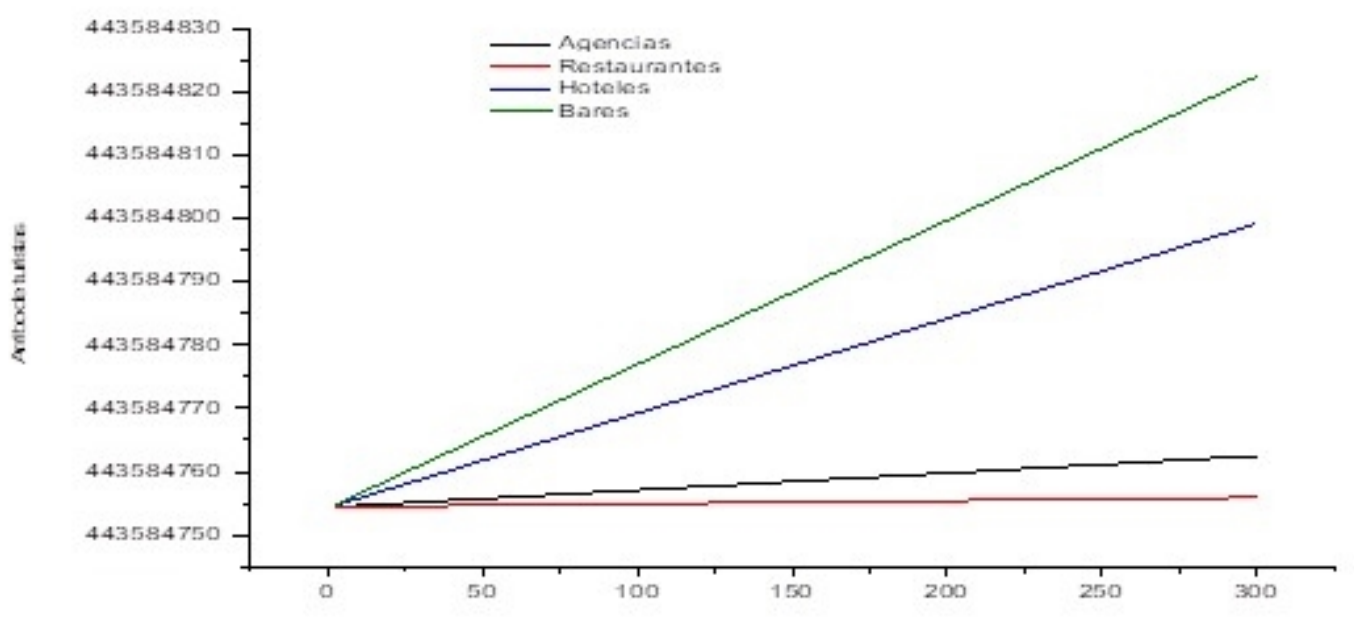

Fuente: Elaboración propia 
1.Esto permite asumir que es preferible para los visitantes escoger hoteles de mayor clasificación turística, en estos destinos los turistas rechazan los hoteles de 1 estrella. Estos datos corroboran la importancia de incentivar y vigilar la mejora en las instalaciones de hospedaje en las zonas turísticas. En el caso de los Turistas que arriban a los lugares de destino de interior estos se relacionan positivamente con los establecimientos de 5, 4, y 1 estrellas, así como con los que no cuentan con una clasificación turística; también existe una relación positiva con los Establecimientos de Alimentos y Bebidas y las Discotecas y Centros Nocturnos. Sin embargo, los Turistas que arriban a los destinos de Interior se relacionan negativamente con los establecimientos de 2 estrellas. Al igual que para el caso de los destinos de playa, el hospedaje juega un papel preponderante en la llegada de turistas a este tipo de destinos, pero también, depende del tipo de turistas y por ende, de su poder adquisitivo. En el caso de los turistas que arriban a los destinos de frontera esto se relacionan positivamente con los establecimientos por clasificación turística de 3 estrellas y sin clasificación turística; así como las empresas arrendadoras de automóviles.

2. Mejora en los servicios de transporte entre y en los destinos turísticos. Una línea de acción para el fomento de los destinos turísticos es la inversión y regulación del funcionamiento de los servicios de transporte dentro y hacia las zonas turísticas. En el caso de México existe un avance significativo en la conectividad aérea y carretera entre los destinos turísticos.
E1 transporte aéreo ha logrado un estándar de calidad. Sin embargo, existen diferencias notables en la calidad y cantidad de los servicios de transporte a los destinos turísticos y dentro de los mismos destinos. Algunos lugares cuentan con terminales de transporte que recientemente han sido renovadas, pero en muchos casos las instalaciones son viejas y en mal estado. Cuando los turistas tienen que viajar a estos destinos utilizando este tipo de transportes tienen que enfrentar servicios caros, localizados en zonas peligrosas o descuidadas, de mala calidad y con pocas o una única oferta. Por otro lado, el transporte dentro de las áreas turísticas constituye un problema grave que ha sido desatendido por las autoridades: los servicios de taxis o los transportes colectivos están en mal estado, el servicio es caro, de poca calidad, con una oferta es limitada y que no cuentan con emisión de comprobantes fiscales. Las autoridades locales y estatales no ejercen funciones de regulación sobre los prestadores de estos servicios, esto ha ocasionado que las tarifas sean fijadas a discrecionalidad sin ninguna proporción entre el tiempo de servicio y el precio.

\section{Conclusiones generales del estudio}

El objetivo del presente documento fue conocer los factores que explican el comportamiento de la demanda turística de 30 lugares de destino en un periodo que abarca de 2000 a 2013 en términos de fomento turístico, utilizando un modelo de regresión múltiple. 
$\mathrm{Al}$ respecto se encontró que las variables independientes mostraron valores significativos, poder explicativo alto, con coeficientes de magnitud considerable y con signos tanto positivos como negativos, lo cual indica que algunas variables guardan una relación directamente proporcional con la variable llegada de turistas, mientras que

otras inciden de manera inversa (negativa) en su comportamiento.

Los resultados obtenidos en el modelo general muestran un poder explicativo del 84 por ciento de las variables incluidas en el modelo. Al respecto se observa que la llegada de turistas está influenciada de manera directa con las agencias de viajes, los restaurantes, los hoteles, los bares y la ocupación hotelera.

Lo anterior pone en relieve la importancia que tiene la disponibilidad de espacios de alojamiento (número de hoteles), pero también, y considerando que un número importante de turistas acude a los diferentes destinos con fines de ocio, recreación y esparcimiento, de la disponibilidad de bares o de agencias de viajes a las cuales los turistas pueden solicitar planes o recorridos específicos, una vez que han llegado. Por su parte, los moteles, las discotecas y centros nocturnos y las cafeterías muestran una relación negativa, lo cual no necesariamente sugiere que el incremento en infraestructura en este tipo de servicios reduce la llegada de turistas al lugar, antes bien, puede significar que estos pueden actuar como bienes inferiores, o que los lugares en los que se hospedan los turistas ya cuentan con este tipo de servicios - (lo cual es muy habitual en los destinos de playa donde en los lugares de alojamiento existen casinos, cafeterías y bares).

Desafortunadamente y debido a que no se cuenta con información desagregada sobre las características de estos servicios, además de que tampoco existe información sobre el perfil de quienes visitan los destinos, las razones por las cuales se establece este tipo de relación con la variable de interés, la interpretación de estos coeficientes debe ser considerada con cautela.

Recapitulando, la implementación de las estrategias y líneas de acción resultarán relevantes para incrementar la llegada de turistas a cada tipo de destino si los montos o apoyos priorizan la creación y generación de infraestructura en materia hotelera, de agencias de viajes, bares y, en menor medida, restaurantes.

\section{Bibliografía}

Guzmán Soria, E., Garza Carranza, M. T., Rebollar, R. S., Hernández Martínez, J., \& García Salazar, J. (2011). Factores determinantes de la demanda internacional del turismo en México. (G. University, Ed.) Globalilzación, competitividad y gobernabilidad. Volumen 5, número 3, 01 de Noviembre: 30-49.

Instituto nacional de estadística y geografía (INEGI). 2000- 2014. Anuario Estadístico y Geográfico. Ediciones de: 1) Acapulco; 2) Cancún; 3) Rivera Maya; 4)Veracruz -Boca del Río; 5) Mazatlán; 6) Puerto Vallarta; 7) Los Cabos; 8) Nuevo Vallarta; 9) Ensenada; 
10) Ixtapa-Zihuatanejo; 11) Manzanillo; 12) Cozumel; 13) Puebla; 14) León; 15) Mérida; 16) Querétaro; 17) Oaxaca; 18) San Juan de los Lagos; 19) Morelia; 20) Tuxtla Gutiérrez; 21) San Luis Potosí; 22) Cuernavaca; 23) Villahermosa; 24) Hermosillo; 25) Xalapa; 26) Guanajuato; 27) Aguascalientes; 28) Zacatecas; 29) Ciudad Juárez; 30) Tijuana. Guerrero", Guerrero. México.

Lancaster K.J. 1966. "A new approach to consumer theory", Journal of Political Economy. 74, pp. 132-157.

McFadden, D. 1973. Conditional Logit Analysis of Qualitative Choice Behavior. In P. Zarembka. ed., Frontiers in Econometrics. Academic Press. Nueva York, 1973.

Morley C.L. (1992): "A Microeconomic theory of international tourism demand", Annals of Tourism Research. 19, pp. 250-267.

Organización Mundial de Turismo (OMT). 2015. Panorama OMT del turismo internacional. Organización Mundial de Turismo. [consulta: 3 de septiembre de 2016]. Disponible en: http://www.eunwto.org/doi/pdf/10.18111/978928441 6875

Rodríguez Feijoo, S., Dávila Quintana, D., \& Rodríguez Caro, A. "Características determinantes del turismo en las islas canarias". Anales de Economía Aplicada, número18. 22-23 de Junio de 2000.
Rugg, D. (1973): "The choice of journey destination: a theoretical and empirical analysis", The Review of Economics and Statistics. 55, pp. 64-72.

Secretaría de Turismo (Sectur). 2013. Programa Sectorial de Turismo 20132018. Diario Oficial de la Federación. 13 de diciembre de 2013. [Consulta: 25 de agosto de 2016). Disponible en: http://www.dof.gob.mx/nota_detalle. php?

codigo $=5326572 \&$ fecha $=13 / 12 / 2013$ 